\title{
Physical Human Interactive Guidance: Identifying Grasping Principles From Human-Planned Grasps
}

\author{
Ravi Balasubramanian, Ling Xu, Peter D. Brook, Joshua R. Smith, and Yoky Matsuoka
}

\begin{abstract}
We present a novel and simple experimental method called physical human interactive guidance to study humanplanned grasping. Instead of studying how the human uses his/her own biological hand or how a human teleoperates a robot hand in a grasping task, the method involves a human interacting physically with a robot arm and hand, carefully moving and guiding the robot into the grasping pose, while the robot's configuration is recorded. Analysis of the grasps from this simple method has produced two interesting results. First, the grasps produced by this method perform better than grasps generated through a state-of-the-art automated grasp planner. Second, this method when combined with a detailed statistical analysis using a variety of grasp measures (physics-based heuristics considered critical for a good grasp) offered insights into how the human grasping method is similar or different from automated grasping synthesis techniques. Specifically, data from the physical human interactive guidance method showed that the human-planned grasping method provides grasps that are similar to grasps from a state-of-the-art automated grasp planner, but differed in one key aspect. The robot wrists were aligned with the object's principal axes in the human-planned grasps (termed low skewness in this paper), while the automated grasps used arbitrary wrist orientation. Preliminary tests show that grasps with low skewness were significantly more robust than grasps with high skewness (77-93\%). We conclude with a detailed discussion of how the physical human interactive guidance method relates to existing methods to extract the human principles for physical interaction.
\end{abstract}

Index Terms-Grasping, haptic interfaces, human robot interaction, manipulators, telerobotics.

\section{INTRODUCTION}

$\mathbf{F}$ OR a roboticist, the way a human grasps or manipulates an object is of great interest for at least two reasons. First, automated grasp planning is still not robust enough when implemented on a physical robot, in stark contrast with human grasps which rarely fail. Second, a personal robotic assistant that uses human-like grasps may perform better in situations

Manuscript received May 2, 2011; revised December 21, 2011; accepted February 22, 2012. Date of publication April 3, 2012; date of current version August 2, 2012. This paper was recommended for publication by Associate Editor L. Villani and Editor J.-P. Laumond upon evaluation of the reviewers' comments.

R. Balasubramanian is with Oregon State University, Corvallis, OR 97331 USA (e-mail: ravi.balasubramanian@oregonstate.edu).

L. Xu is with Carnegie Mellon University, Pittsburgh, PA 15213 USA (e-mail: lingx@andrew.cmu.edu).

P. D. Brook is with the University of Washington, Seattle, WA 98195 USA, and also with Facebook, Menlo Park, CA 94025 USA (e-mail: pbrook@cs.washington.edu).

J. R. Smith and Y. Matsuoka are with the University of Washington, Seattle, WA 98195 USA (e-mail: jrsintel@ gmail.com; yoky@ alum.mit.edu).

Color versions of one or more of the figures in this paper are available online at http://ieeexplore.ieee.org.

Digital Object Identifier 10.1109/TRO.2012.2189498 when the human and the robot comanipulate an object. For example, when a robot is handing an object to a human, it would be better if the robot grasped the object proximally rather than distally so that the person can grasp it. However, there is still much to learn about the heuristics that make human grasping or even human-planned grasping (a grasp that is planned by a human, say, for a robot) so robust. This paper presents a novel experimental method that enables a direct comparison of humanplanned grasping with the performance of a state-of-the-art automated grasp planning algorithm and simultaneously identify the heuristics humans use in performing grasps. In particular, this paper presents a previously unnoticed grasping heuristic called skewness, which significantly improves robotic grasping performance as well.

Apart from direct observation of humans using their hands [12], two primary approaches have been popular in the literature to identify how humans perform grasps: 1) Performing motion capture of the human hand itself when performing grasps using vicon cameras [30], [34], data gloves [16], [27], [39], force sensors [48], or video footage [4], [11]; 2) studying the grasps that humans plan for the robot using teleoperation either through direct sight [10], [37], using real-time video [14], [26], [29], or using a virtual environment [24].

However, there are significant challenges with these approaches. First, the human hand's complex geometry makes a direct study of its posture in grasping experiments very challenging. While the exact numbers are debated, the human hand has over 21 degrees of freedom (DOF), including joints in the fingers, thumb, palm, and wrist [22]. In addition, the joints's rotational axes are, typically, nonorthogonal and nonintersecting and, usually, differ between human subjects [5], [6], [17], [44]. Finally, the high compliance of the palm and skin and feedback control loops [20], [45] in the human body make grasp contact analysis difficult. Consequently, the large parameter space and the approximations made in describing hand kinematics and contact complicate the identification of the heuristics behind finger and wrist posture in a human grasp [11], [30]. Furthermore, if we want to use the human grasping heuristics to improve the performance of robotic grasping, then the difference in kinematics between the human hand and the robotic hand poses a further challenge. For example, the popular BarrettHand ${ }^{1}$ [43] has only four joints compared with the many joints in the human hand. There is no straightforward procedure to map the human hand posture to the robot hand, and consequently, human hand grasps to robotic grasps (see [19] for an example).

Similarly, there are challenges in extracting the principles of human grasping from the human-planned grasps obtained

\footnotetext{
${ }^{1}$ http://www.barrett.com/robot/index.htm
} 


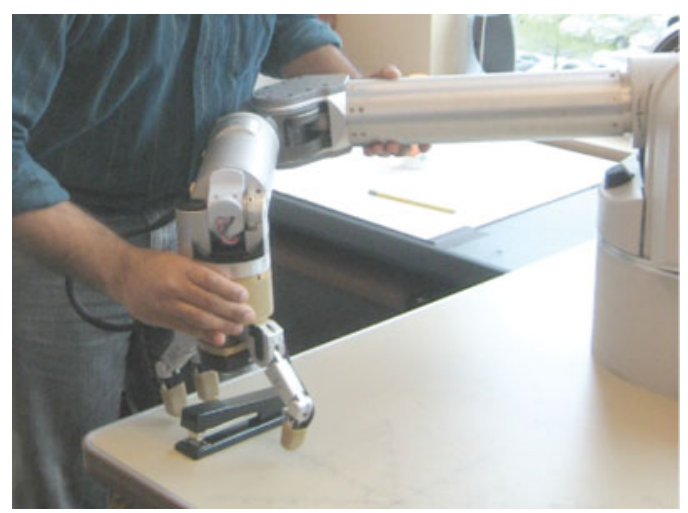

Fig. 1. Physical human interactive grasping environment. The human physically guides the robot wrist and fingers into a grasping posture.

through teleoperation. The artificial (and usually) 2-D visual or haptic feedback that the human is provided may limit the human subject's choice of grasps. Thus, the human may provide suboptimal grasps arising from poor access. In addition, the more physically removed the human is from the task, the role of practice and training becomes more significant to achieve optimal performance. Thus, there may be strong variability in the grasps that the human subjects provide, depending on the variability in experience operating the device. As a result of these challenges, these works have primarily yielded qualitative information about human grasping, such as a grasp taxonomy [12] and the postural synergies in hand grasping movements [39], from which it is difficult to infer which aspects of human grasping lead to its high robustness.

To achieve our goal of identifying human grasping principles, we wanted a data collection process that allowed the human to express their grasping intentions naturally with minimal training. Simultaneously, we wanted an easy and straightforward method to interpret the kinematics of the human grasp.

Our approach, in contrast with existing techniques, allowed the human subjects to plan a grasp for the robot by physically guiding the robot hand (wrist and fingers) into a grasping posture for a given task (see Fig. 1). This procedure, which is called physical human interactive guidance, allowed the human subject to be, intimately, involved in the task - arguably as involved and simple as placing a pair of tongs on an object for grasping. The advantage of the human subject using the robot hand rather than his/her own hand is that the geometry of robot wrist and finger placement is straightforward to measure through the joint encoders in the robot. Another unique aspect of our method is that instead of a standalone analysis of human-planned grasping, since the human plans grasps for a robotic hand, we can compare the human-planned grasps with grasps generated for the same robot by an automated grasp planner [42].

We first verified that the grasps collected using the physical human interactive guidance method performed better than grasps generated for the robot by a state-of-the-art automated grasp planning software, even though both the human and the automated planner provided the same information to the robot, namely, wrist orientation and finger posture (and no dynamic information such as contact force). Second, we showed that even though the subjects were not using their biological hands to perform grasps, we could still identify critical heuristics that humans use in grasping by comparing the human-planned grasps with the grasps from the automated planner, including a new human grasping principle that to our knowledge has not been noticed earlier and that significantly improves grasping performance on a robot when used to filter automated grasp planning results.

After describing our method for collecting human-planned grasping data in Section II and the method for testing the grasps in Section III, we, then, present a method to analyze the humanplanned grasps in Section IV. Section V provides the results of the experiments conducted, in terms of the success rate of the human-planned grasps and the key parameters optimized by the human-planned grasps. We also show how the physical human interactive guidance method identified a new grasp measure. In Section VI, we discuss how this novel method for grasp acquisition relates to previous methods in the context of human-robot interaction. Portions of this study were, briefly, reported earlier in [1], but that paper did not focus on the novelty of the data collection method. In addition, this paper provides additional insights into human-planned grasping heuristics and the effectiveness of human involvement in teaching robots.

\section{Physical Human Interactive Guidance}

Our approach to acquire grasping examples from humans allowed a human subject to teach a robot different grasps by being in the robot's workspace and physically interacting with the robot. This interaction method required the person to guide the robot to specific wrist configurations and finger postures. This experimental setup was called the physical human interactive guidance environment (see Fig. 1) and the grasps collected "human-planned grasps."

Through the physical human interactive guidance method, the human subject had an opportunity to understand the motion capabilities of the robot arm and hand, the object's inertial and geometrical properties, and how the robot and object would interact during the grasp (including the type of contact). These aspects of the grasping process are critical since grasping is a physically interactive task where the "last few centimeters" could make the difference between a successful and unsuccessful grasp. That is, however carefully the hand's path was planned to reach the grasping posture, the grasp could still fail if the finger placement was not good. Note also that the human subject could move freely around the robot to use different views of the object-hand interaction to decide on the best grasp posture (in contrast with other work that has explored how limited visual feedback can affect human grasping [8] and reaching [47] strategies). The physical human interactive guidance method was possibly the most intimate way for human subjects to build an internal model of the grasping process using a robot hand.

Such interactive robotic grasping with a human in the loop has been explored before by the GraspIt! group [9], but their goal and approach was different from the study in this paper. Their goal was to demonstrate how GraspIt! goes through search 
iterations to generate a grasp for a given wrist position. In addition, only wrist posture was controlled by the human and finger posture was controlled by GraspIt!. The purpose of our experiment was to identify what was unique about human-planned grasping strategies, which might not be expressed properly in other methods. In addition, in our method, the human had full control over the wrist and finger posture, both of which are critical to grasp quality.

\section{A. Robotic Equipment}

The physical human interactive guidance environment used a robotic platform consisting of a 7-DOF Barrett whole arm manipulator robotic arm and a three-fingered 4-DOF BarrettHand $^{2}$ [43]. The robotic system was run on Willow Garage's ROS software, ${ }^{3}$ and the robotic hand was equipped with electric field sensors [46] which enabled the fingers to detect their proximity to objects. The electric field sensors were used, primarily, to close the fingers on the object simultaneously. Note that the choice of robotic equipment used in this paper is only incidental to available resources, and other robotic arms and hands could be used to recreate the experimental setup.

\section{B. Grasp Guidance and Acquisition}

Grasp data acquisition was kept as simple as possible. The object to be grasped was placed by the experimenter at a known location and orientation in the robot's workspace. The robot arm was placed in a "gravity compensation" mode, where the robot arm had negligible weight and could be easily moved by a human subject. The robot arm was reset to a neutral position in the workspace and the robot hand's fingers were kept open. The grasp guidance procedure included the following four steps.

Step 1: The human subject physically guided the robot arm to an initial wrist pose at which the object could be grasped [see Fig. 2(a) and 2(b)]. The human subject was free to move around the robot and use as many views as necessary to position the robot wrist with or without an offset with respect to the object axes.

Step 2: Using electric field sensing, the finger motors were commanded to close on the object so that each fingerpad was approximately $5 \mathrm{~mm}$ from object surface. At this point, the BarettHand motors were turned OFF to allow the human subject to physically adjust the spread angle of the fingers, depending on whether a parallel gripper-like grasp or a three-finger triangular grasp was desired. Additionally, the subject could adjust wrist pose again to better align the fingers with the object [see Fig. 2(c)]. Importantly, the human subject was again given ample freedom, time, and space to move around the robot in order to choose what he/she believed was the best grasp for that task.

Step 3: When the subject was satisfied with this grasp pose, the robotic fingers were commanded to close on the object, completing the grasp guidance procedure. The final closure step was guided by the electric field sensors so that all fingers contacted at the same time as to not perturb the object [see Fig. 2(d)].

\footnotetext{
${ }^{2}$ http://www.barrett.com/robot/index.htm

${ }^{3}$ http://www.ros.org/
}

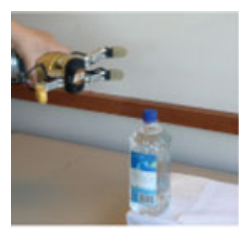

(a)

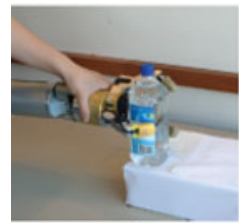

(d)

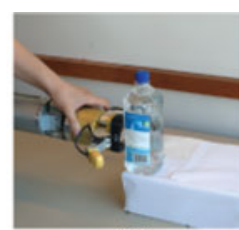

(b)

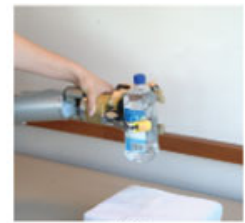

(e)

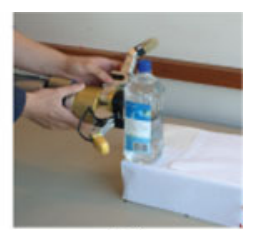

(c)
Fig. 2. Experimental procedure of a human subject guiding the robot to grasp an object: (a) and (b) Approach the object, (c) adjust wrist orientation and finger spread, (d) fingers close in on the object, and (e) lift object. Note that the subject was free to move around the workspace to view the physical interaction from multiple angles.

Step 4: Subjects were, then, allowed to lift and shake the robotic arm to determine if they liked the grasp. Note that this light shaking performed by the subject is different from the vigorous programmed shaking that was performed during the grasp testing phase (see Section III). If the subject did not like the grasp or if the object slipped out, the grasp was disregarded [see Fig. 2(e)]. We eliminated such grasps because the goal was to collect the best grasps that humans could provide.

Since the subjects had less than 5 min of practice with the system before experiment data were collected, the grasp guidance procedure provided an opportunity for the subjects to review the grasps. This allowed the subjects to understand the grasping process with the robotic hand and build an internal model based on their grasping experiences with their own hands. It turned out in the experiment described in the next section that less than $5 \%$ of all the human guidance grasps were eliminated because the subject was not satisfied with the grasp. Therefore, the grasp review process did not, significantly, affect the set of grasps collected.

Each grasp was represented simply as the kinematic configuration of the robot arm and hand relative to the object reference frame. Thus, a grasp was an 11-D vector containing the 7-DOF robot arm joint angles and the 4-DOF hand joint angles (one spread and three flexion) relative to the object's reference frame.

\section{Human-Subject Experiment Paradigm}

Seven human subjects participated in a study approved by the University of Washington Human Subjects Division. Each subject was given $\mathrm{f} 5 \mathrm{~min}$ of practice with the robot, and a total of 210 grasps were collected with the robot. Nine everyday objects were used in the experiment: Three small objects, three medium-sized objects, and three large objects (see Fig. 3).

Since these everyday objects had straightforward geometry, the experimenter used the objects's edge features to, carefully, position the objects at the required location and orientation with 


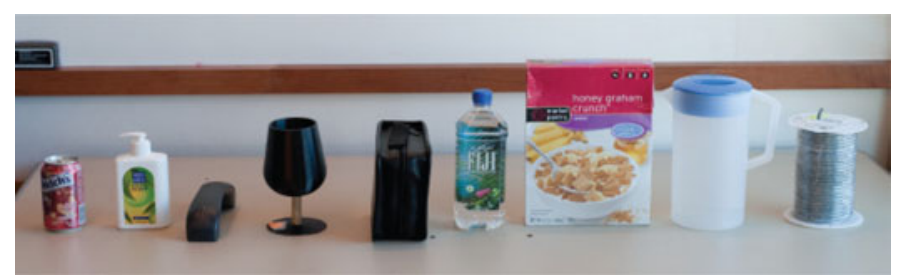

Fig. 3. Objects used in the experiment fall into three size categories: Small, medium, and large.

TABLE I

FUNCTIONAL TASKS

\begin{tabular}{c|c}
\hline Object & Functional task \\
\hline Wine glass & Lift to pour \\
One-liter bottle & Lift to pour \\
Soda can & Lift to pour \\
Cereal box & Lift to pour \\
Coil of wire & Lift to remove wire \\
Phone & Lift to answer \\
Pitcher & Lift to pour \\
Soap dispenser & Lift to dispense \\
CD pouch & Lift to open \\
\hline \hline
\end{tabular}

respect to a known world coordinate frame marked on the table. ${ }^{4}$ Since the experiment was to study the details of human-planned grasping of everyday objects in natural configurations, the objects were placed in the vertical orientation only (as shown in Fig. 3). Since the robot base's position and location was also known accurately with respect to the world coordinate frame and the robot's forward kinematics available from the manufacturer, the robot hand's pose could be computed in the object's frame of reference. ${ }^{5}$

Each subject was asked to perform three different tasks for an object, namely, lifting the object, handing the object over, and performing a function with the object. For the handing over task, the subject was asked to grasp the object such that there was space left for someone else to grasp it. The functional tasks depended on the object. For example, the functional task for the wine glass was pouring and for the phone, the task was picking up to make a phone call (see Table I for complete list).

For each object-task pair, the subject was asked to provide two grasps, providing a total of six grasps per object. The subjects were asked to vary the grasps if they could so as to obtain some variety in the grasps collected. Each subject was randomly assigned to five objects, while ensuring an even distribution of grasps for each of the objects (each object was selected four times except for the soda can that was selected three times).

\footnotetext{
${ }^{4}$ In this particular experiment (see Fig. 2), a white rectangular box on which the objects were placed was used to align the object. This was only incidental to this experimental setup, and any means of repeated accurate positioning of the object will suffice.

${ }^{5}$ We also placed the objects, randomly, in three different locations on the table (left, right, and center with respect to the robot base) to ensure that the human-planned grasps were not unduly influenced by the specificity of the arm posture required for a particular location. Since we did not find any significant differences between the grasps from different locations in terms of the robot wrist and finger posture relative to the object, we combined all the humanplanned grasps from the different locations into one set to be tested by the stationary robot.
}

After each human-planned grasp, the object was again placed carefully by the experimenter in the required position and orientation and the experiment was repeated.

The human subjects also responded to a questionnaire to help identify the heuristics they believed they used to perform the grasping task. Specifically, we wanted to find out what geometric and force-related aspects of the grasp the subjects thought they used to perform the grasp. For example, one specific question was asked if the subjects paid attention to wrist orientation and finger posture (geometric) and wrist position relative to object center of mass (force-related). More details are presented in Section V.

\section{Grasp Testing ON Physical Robot}

After collecting the human-planned grasps, we wanted to test how well each grasp performed on average on those same objects. Several past works have tried to infer grasp quality simply from simulation models [3], [15], [25], [28], [32], [33], [36], [42] with mixed results (see Section V). In this paper, the human-planned grasps were validated on a physical robot rather than in simulation. From the eight human-planned grasps for each object-task pair (six for the soda can), our protocol was to choose three grasps, randomly, for testing on a physical robot. Thus, we expected to test a total of 27 grasps for each task (three candidate grasps $\times$ nine objects). However, it turned out that some human-planned grasps for the lifting and functional task, which were performed when the objects were placed to the left and right of the robot, could not be tested on the stationary robot when the grasps were mapped to the center location (in particular, grasps from the object's front) due to the lack of an inverse kinematics solution. Thus, all the human-planned grasps for the lifting and functional tasks, which could be tested ( 25 grasps), were tested.

The testing procedure was, intentionally, kept simple. The object was placed by the experimenter in a known position and orientation (similar to the procedure outlined in Section II-C). Since each grasp was represented as an 11-D vector of robot arm and hand joint angles relative to the object, the robot was simply commanded to the grasp posture as follows. The robot arm was commanded to the recorded arm joint angles with the fingers fully opened. The robot hand was, then, commanded to the required spread angle. Finally, the fingers were commanded to close in quickly on the object, and the robot lifted the object and, then, executed a shaking procedure, where the object was shaken by the robot four times in a continuous circular motion (see Table II for peak and mean velocities and accelerations). Note that this automated shaking by the robot was different and significantly more vigorous than the light shaking that the users performed after they planned the grasp (see Step 4 in Section II-B). In addition, the users did not know that the grasp would be tested in this manner.

If the object stayed in the hand after the shaking, it was considered a success (scored 1). All other situations (object pushed away during acquisition or object falls down during shaking) were considered a failure (scored 0 ). This testing process was repeated for each grasp five times. 
TABLE II

SHAKING TRAJECTORY DETAILS

\begin{tabular}{c|c|c}
\hline & Peak & Mean \\
\hline Angular Velocity $(\mathrm{rad} / \mathrm{s})$ & 4.62 & 2.74 \\
Linear Velocity $(\mathrm{m} / \mathrm{s})$ & 0.62 & 0.39 \\
Angular Acceleration $\left(\mathrm{rad} / \mathrm{s}^{2}\right)$ & 4.39 & 2.22 \\
Linear Acceleration $\left(\mathrm{m} / \mathrm{s}^{2}\right)$ & 0.63 & 0.33 \\
\hline \hline
\end{tabular}

Note that this simple grasp testing procedure helps maintain the focus of our research on grasp generation rather than elaborate grasp testing methods that include, say, feedback control. The success rate was computed for each grasp by averaging over the five trials. Hypothesis testing was performed with a significance level of 0.05 , and standard errors were reported for all mean values.

\section{GRASP ANALYSIS}

\section{A. Analysis Using a Grasp Measure Set}

Given our goal of identifying the principles behind humanplanned grasping, we needed a grasp measure space that identified the properties of a grasp. Specifically, the 11-D finger and wrist posture of the grasp alone does not provide insights into grasp quality, since the way the fingers are placed relative to the object is critical for the grasp. Several grasp measures have been proposed in prior literature to infer grasp quality [2], [7], [15], [31], [35], [38], [40]. After a detailed survey, we chose a set of 11 grasp measures from the literature (see Table III; the citations correspond to all the features in each section of the table). The "grasp volume" measure is a 3-D version of the "grasp area" suggested for planar grasps in [7].

Each grasp measure is associated with a heuristic. For example, the epsilon metric in row 1 of Table III measures the minimum disturbance force and moments that a grasp can resist in all directions, and results from simulation show that a grasp is better if it has a larger epsilon score [15]. But few grasp measures have been, rigorously, evaluated using experiments on a physical robot.

The last row in Table III also proposes a new grasp measure that was suggested by the data during the human-subject experiments and that we hypothesize may be broadly useful. The new measure, which is called skewness, measures the robot wrist orientation relative to the object's principal axis. Suppose the object's principal axis (axis of longest dimension) is represented by unit vector $u$, and the axis pointing out of the palm of the BarrettHand by unit vector $v$ (see Fig. 4). The angle $\delta$ between $u$ and $v$ may be computed as $\delta=\arccos (u \cdot v)$. Then, the skewness measure $\alpha$ is defined as

$$
\alpha= \begin{cases}\delta, & \text { if } \delta<\pi / 4 \\ \pi / 2-\delta, & \text { if } \pi / 4<\delta<\pi / 2 \\ \delta-\pi / 2, & \text { if } \pi / 2<\delta<3 \pi / 4 \\ \pi-\delta, & \text { if } \delta>3 \pi / 4\end{cases}
$$

In the human-planned lifting grasp for the bottle in Fig. 5, robot's wrist orientation in the bottle-lifting task is approximately parallel to the bottle's principal axis (vertical), and the

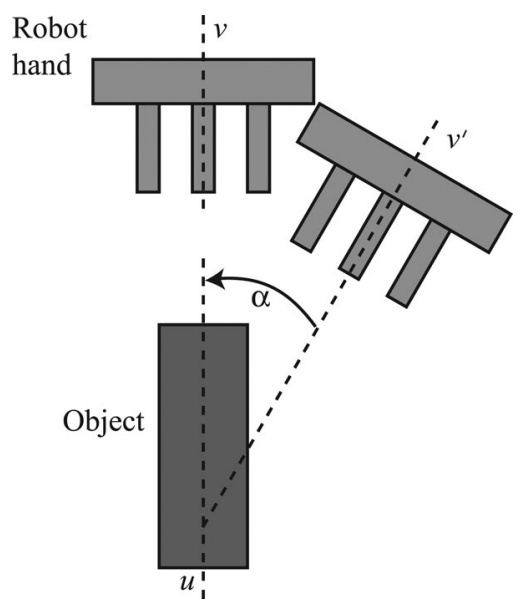

Fig. 4. Relative orientation of the object and robot hand: The object's principal axis is $u$. In pose $v$, the robot hand has skewness of zero, while in pose $v^{\prime}$, the robot hand has skewness close to $30^{\circ}$.

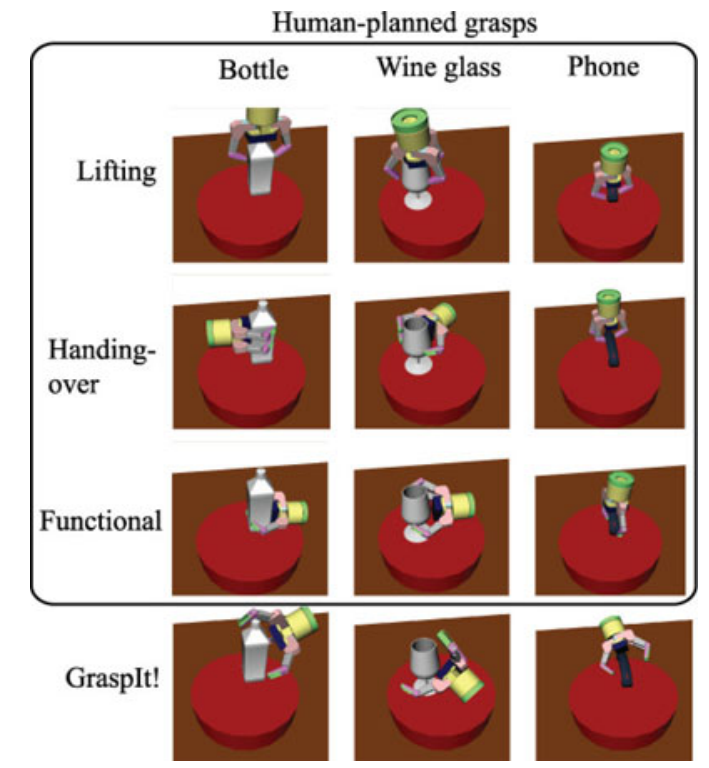

Fig. 5. Example grasp postures generated by human subjects (for a lifting task) and GraspIt! for three objects. Note that the human subjects, manually, specified the grasps on the physical Barrett robotic hand, which were then visualized using the OpenRAVE program [13].

grasp's skewness measure $\alpha$ is near zero. In contrast, the computer generated GraspIt! grasp for the bottle would have a skewness measure $\alpha$ close to $30^{\circ}$.

Note that it was easy to notice the peculiarity of wrist placement in the human-planned grasps only because we used the physical human interactive guidance method. This is because the robot hand geometry is simple and known explicitly, and the subjects were comfortable with the guidance process. Thus, the subjects were able to use their natural grasping heuristics, and we could identify the new skewness measure. In contrast, if we were studying the human hand directly, it would have been significantly more difficult to identify a grasp measure, such as skewness, due to the complexity of the human hand geometry.

We computed values for all the grasp measures for each human-planned grasp, and thus, the grasp could now be 
TABLE III

GrasP MEASURE SET

\begin{tabular}{|c|c|c|c|c|}
\hline Grasp Measure & Description & Minimum & Maximum & Citation \\
\hline Epsilon $^{a}$ & $\begin{array}{l}\text { Minimum disturbance wrench that } \\
\text { can be resisted }\end{array}$ & 0 & 1 & $\begin{array}{l}{[15],} \\
{[31]}\end{array}$ \\
\hline Wrench space volume ${ }^{a}$ & Volume of grasp wrench space & 0 & $2^{6}$ & \\
\hline Grasp energy ${ }^{b}$ & Hand-object proximity & Negative infinity & Infinity & \\
\hline Point arrangement ${ }^{a}$ & $\begin{array}{l}\text { Proximity of fingertips being in a } \\
\text { plane parallel to palm. }\end{array}$ & 0 & 1 & [7] \\
\hline Grasp volume $^{a *}$ & Volume enclosed by hand & 0 & $\sim 669 \mathrm{~cm}^{3}$ & \\
\hline Hand flexion ${ }^{b}$ & Similarity of finger flexion & 0 & 1 & \\
\hline Hand spread ${ }^{b}$ & $\begin{array}{l}\text { Proximity of the finger spread to equi- } \\
\text { lateral triangle }\end{array}$ & 0 & 1 & \\
\hline Finger limit ${ }^{c}$ & Extent of finger extensions & 0 & 1 & \\
\hline Volume of object enclosed ${ }^{a}$ & $\begin{array}{l}\text { Object volume enclosed by hand nor- } \\
\text { malized by object volume }\end{array}$ & 0 & 1 & $\begin{array}{l}{[41],} \\
{[40]}\end{array}$ \\
\hline Parallel symmetry ${ }^{b}$ & $\begin{array}{l}\text { Distance between center of mass and } \\
\text { contact point centroid along object } \\
\text { principal axis }\end{array}$ & 0 & 0.5 & \\
\hline Perpendicular symmetry ${ }^{b}$ & $\begin{array}{l}\text { Distance between center of mass and } \\
\text { contact point centroid perpendicular } \\
\text { to object principal axis }\end{array}$ & 0 & 0.5 & \\
\hline Skewness & See section IV-A & 0 & $\pi / 4$ & \\
\hline
\end{tabular}

${ }^{a}$ Larger $\Rightarrow$ Better grasp; ${ }^{b}$ Smaller $\Rightarrow$ Better grasp; ${ }^{c}$ Mid-range $\Rightarrow$ Better grasp.

evaluated in the chosen 12-D grasp-measure space. This will help understand the parameters that humans optimize for when performing grasps. Note that this is in addition to testing on a physical robot, which provides a true measure of grasp quality.

\section{B. Comparison With Automated Grasping Methods}

Instead of a stand-alone analysis of human-planned grasping, we wanted to compare the human-planned grasping technique with existing grasp synthesis methods both in terms of average success rate as well as the heuristics optimized for during grasp generation. The most common and standardized procedure in the robotics community is automated grasp synthesis for robots using a set of grasp measures. We used an open-source stateof-the-art grasp planning software called GraspIt! developed by Columbia University [32] for grasp generation. ${ }^{6}$ Note that we could have used other software such as OpenRAVE [13] as well for grasp generation. However, many of these programs use the same standard force-closure metrics developed by the robotics community to evaluate grasp quality [15]. We chose GraspIt! since it had been well tested for several years and their team helped us understand the code.

Given an object's 3-D model, GraspIt! finds grasps for an object by searching the high-dimensional hand-configuration space and, then, focuses the search on the best grasps by using a variety of grasp measures. Combined with a compliant contact (soft-contact) simulation, GraspIt! uses the following grasp measures that are popular in the robotics community: 1) Wrench space computations (epsilon and volume [15]) that estimate a grasp's ability to provide force closure based on the minimum disturbance that the grasp can resist in all directions (first and second rows in Table III); and 2) the shortest distance between the object and predefined grasp points on the hand (defined as grasp energy in the third row of Table III).

Using the same procedure that was used to generate grasps for the Columbia Grasp Database [18], we ran GraspIt! for

\footnotetext{
${ }^{6}$ http://grasping.cs.columbia.edu/
}

30 min with the intention of generating multiple top grasps for each object according to its grasp heuristics. In $30 \mathrm{~min}$, GraspIt! explored a large set (135 000) of varied wrist and finger configurations to generate six top grasps for most of the objects, but for three objects, GraspIt! generated only four or five grasps (wine glass: 4, coil of wire: 5, 1-L bottle: 4). This was, partly, due to search complexity as well as the lack of an inverse kinematics solution when implemented on the robot (since the robot was stationary relative to the table and object in the setup). Thus, the automated grasp search provided a total of 49 grasps across the nine objects after exploring 1.2 million wrist and finger configurations. Since we collected a sufficient number of automated grasps from GraspIt!, we did not feel the few grasps that we lost to the search complexity were significant in our results. Note again that each grasp is represented as the $11-\mathrm{D}$ vector containing robot arm and hand joint angles.

Note that GraspIt! cannot provide task-specific grasps, and its grasps are intended for lifting tasks only. Therefore, the performance of only the human-planned lifting grasps and GraspIt! grasps will be directly compared. The GraspIt! grasps also were validated using the same process as the human-planned grasps (see Section III).

\section{REsults}

\section{A. Human-Planned Grasps Versus Automated Grasps}

Fig. 5 shows a sample of grasps generated by GraspIt! and through the physical human interactive guidance method for the different tasks. All the fingers were used in every grasp, whether human planned or from GraspIt!.

Table IV presents the success rates for each object (averaged over five trials) for the human-planned grasps and for the GraspIt! grasps (a total of $(25+27+25+49) \times 5=630$ testing trials). Across objects, the human-planned lifting strategy yielded a 91(3)\% success rate, while GraspIt! yielded 77(3)\%. An outlier for the human lifting grasps was the 1-L bottle. If these grasps were removed, the success rate for human-planned 
TABLE IV

MEAN SUCCESS RATES FOR HuMAN-PLANNEd GRASPING AND GRASPIT!

\begin{tabular}{c|c|c|c|c}
\hline Object & \multicolumn{3}{|c|}{ Human-planned } & Grasplt! \\
& Lifting & Handing-over & Functional & \\
\hline Wine glass & $93(7)$ & $33(13)$ & $100(0)$ & $100(0)$ \\
One-liter bottle & $40(13)$ & $67(13)$ & $93(7)$ & $65(26)$ \\
Soda can & $93(7)$ & $87(9)$ & $100(0)$ & $90(5)$ \\
Cereal box & $93(7)$ & $87(10)$ & $100(0)$ & $90(4)$ \\
Coil of wire & $100(0)$ & $100(0)$ & $60(13)$ & $32(11)$ \\
Phone & $100(0)$ & $100(0)$ & $50(13)$ & $70(6)$ \\
Pitcher & $100(0)$ & $100(0)$ & $100(0)$ & $83(5)$ \\
Soap dispenser & $100(0)$ & $87(9)$ & $100(0)$ & $67(11)$ \\
CD pouch & $100(0)$ & $100(0)$ & $67(13)$ & $100(0)$ \\
\hline Overall & $\mathbf{9 1 ( 3 ) *}$ & $\mathbf{8 4 ( 3 )}$ & $\mathbf{8 6}(\mathbf{3})$ & $\mathbf{7 7 ( 3 ) *}$ \\
\hline Number of grasps & 25 & 27 & 25 & 49 \\
\hline \hline
\end{tabular}

$* \Rightarrow p<0.05$ when comparing the human-planned lifting and GraspIt! grasps.

TABLE V

GrasP Measure Values For Human-PlanNed GrasPing AND GraspIT!

\begin{tabular}{|c|c|c|c|c|}
\hline \multirow[t]{3}{*}{ Grasp measure } & \multicolumn{4}{|c|}{ Mean (Standard error) } \\
\hline & \multicolumn{3}{|c|}{ Human-planned } & \multirow{2}{*}{ GraspIt! } \\
\hline & Lifting & Handing-over & Functional & \\
\hline Epsilon & $0.1(0.02)^{*}$ & $0.10(0.01)$ & $0.09(0.01)$ & $0.19(0.01)^{*}$ \\
\hline Wrench space volume & $0.15(0.05)^{*}$ & $0.14(0.03)$ & $0.19(0.06)$ & $0.42(0.04)^{*}$ \\
\hline Grasp energy & $-1.33(0.09)$ & $-1.46(0.07)$ & $-1.2(0.09)$ & $3.95(2.57)$ \\
\hline Point arrangement & $0.78(0.02)$ & $0.79(0.02)$ & $0.75(0.02)$ & $0.76(0.02)$ \\
\hline Grasp volume $\left(\mathrm{cm}^{3}\right)$ & $281(29)$ & $271(24)$ & $238(35)$ & $259(33)$ \\
\hline Hand flexion & $0.05(0.01) * a$ & $0.05(0.01)^{b}$ & $0.29(0.08)^{a, b}$ & $0.19(0.04) *$ \\
\hline Hand spread & $0.39(0.02)$ & $0.38(0.02)$ & $0.43(0.02)$ & $0.37(0.02)$ \\
\hline Finger limit & $0.70(0.05)$ & $0.71(0.02)$ & $0.73(0.03)$ & $0.76(0.02)$ \\
\hline Volume of object enclosed & $0.06(0.01)$ & $0.06(0.01)$ & $0.05(0.01)$ & $0.05(0.01)$ \\
\hline Parallel symmetry & $0.30(0.05)$ & $0.35(0.05)$ & $0.28(0.04)$ & $0.39(0.03)$ \\
\hline Perpendicular symmetry & $0.33(0.03)$ & $0.30(0.03)$ & $0.33(0.04)$ & $0.28(0.03)$ \\
\hline Skewness & $5.2(1.3)^{*}$ & $6.09(1.90)$ & $4.79(1.01)$ & $23.2(1.86)^{*}$ \\
\hline Number of grasps & 25 & 27 & 25 & 49 \\
\hline
\end{tabular}

lifting grasps would be $97(1) \%$. Interestingly, while the humanplanned grasps for the handing over task and the functional task did not perform as well as the human-planned grasps for lifting, they still outperformed, on average, the GraspIt! grasps which are meant for lifting only.

Table $\mathrm{V}$ shows the range of values for the grasp measures for human-planned grasps and the GraspIt! grasps. Looking first at the human-planned lifting grasps and the GraspIt! grasps, we notice that only four grasp measures, namely, epsilon, grasp wrench-space volume, hand flexion, and skewness, were significantly different between the two grasp sets. The energy measure showed a borderline significant difference $(p=0.05)$ between human-planned lifting and GraspIt!, but that was due to outliers.

While larger epsilon and volume indicated better grasp quality theoretically, we noticed from the experiment that epsilon and volume were lower for the human-planned grasps when compared with the GraspIt! grasps, even though the human guided grasps had a higher success rate than the GraspIt! grasps. The hand-flexion measure indicated that humans used lifting grasps which had significantly different finger flexion values when compared with the GraspIt! grasps. The hand-spread values for the human-planned grasps indicated that the humans used largely pinch grasps with low spread. This also led to small volumes of the object enclosed by a grasp.

The stand-out grasp measure, however, was skewness. The skewness measure for the human lifting grasps was significantly smaller than for the GraspIt! grasps, indicating that wrist orientation in the human-planned lifting grasps are much closer to the object's principal axis or its perpendiculars (see Fig. 5;

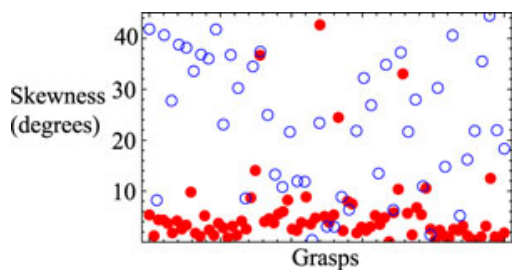

Fig. 6. Scatter plot of the skewness measure of the human-planned lifting grasps (red dots) and the GraspIt! grasps (blue circles)

the principal axis for the bottle and wine glass was vertical and phone horizontal). Fig. 6 shows a scatter plot of the skewness measure for human-planned lifting grasps (mean $5.2(1.3)^{\circ}$ ) and the GraspIt! grasps (mean $23.2(2)^{\circ}$ ), indicating that the human-planned grasps used wrist orientation that deviated very little from the objects's principal axes, whereas the automated grasps' wrist orientations were scattered all over.

Focusing on the task-dependent human-planned grasps, Fig. 5 shows some examples of variation in grasping strategy for different task requirements. Grasps used by the handing over and functional tasks were not statistically different from the liftingtask grasps as measured by these grasp measures except for the hand-flexion measure $(p<0.05)$. The hand-flexion measure showed differences between the functional human-planned grasps and the lifting and handing-over human-planned grasps. This indicated that the functional task caused the human subjects to change the hand flexion significantly.

There were near-significant differences $(0.05<p<0.1)$ between the handing-over and functional human-planned grasps for the finger spread, parallel symmetry, and energy grasp measures, and near-significant differences between the lifting and functional human-planned grasps for the finger-spread grasp measure. Interestingly, the skewness measure was low for the human-planned handing over $\left(6.3(1.8)^{\circ}\right)$, as well as for functional tasks $\left(4.8(1.0)^{\circ}\right)$.

\section{B. GraspIt! Performance Improvement With Low Skewness}

Each grasp, whether from GraspIt! or planned by a human, was stored as an 11-D vector containing the seven robot arm angles and four hand joint angles. All the grasps were divided into two groups: Group 1 was the set of grasps obtained by merging the set of human-planned lifting grasps and the set of grasps from GraspIt!. Group 2 consisted of GraspIt! grasps only. Fig. 7 shows the variation in success rates for the two groups of grasps, each split by a skewness threshold of $13^{\circ}$. This result showed that the success rate of low-skewness grasps from GraspIt! was significantly higher than high-skewness grasps from GraspIt! $(93(5) \%$ compared with $77(3) \% ; p$-value $=0.01)$. In contrast, when investigating the significance of the hand-flexion measure for grasping, we did not see a significant difference in grasp success for grasps with small hand-flexion measures when compared with grasps with large hand-flexion measures. This indicated that a low hand-flexion measure was, likely, not a reason for a better grasp. 


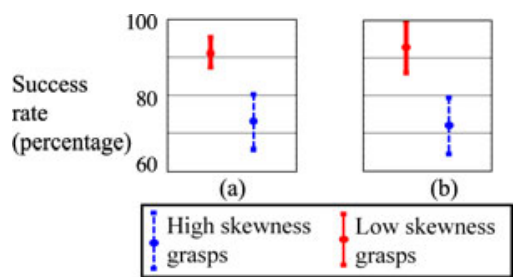

Fig. 7. Success rates for low-skewness $\left(<13^{\circ}\right)$ and high-skewness grasps from two groups. (a) Human lifting grasps combined with GraspIt! grasps (lowskewness and high-skewness grasps $n=37$ each; $p$-value $=0.01$ ). (b) Grasplt! grasps only (low-skewness grasps $n=14$, high-skewness grasps $n=35$; $p$ value $=0.01$.

TABLE VI

HuMAN SUBJECT QUESTIONNAIRE RESULTS

\begin{tabular}{c|c}
\hline Heuristic & Positive Response (\%) \\
\hline Object curves influence grasp & 100 \\
Use finger spread for stability & 100 \\
Grasp close to center of gravity & 86 \\
Object weight influence grasp & 71 \\
Keep robot wrist vertical/horizontal & 71 \\
Ridges influence grasp & 71 \\
Use palm of hand for grasp & 57 \\
Grasp strategy change with practice & 43 \\
\hline \hline
\end{tabular}

\section{Questionnaire}

Table VI provides the results of the short questionnaire provided to the human subjects, the rows ordered in decreasing importance of the heuristic (according to the subject's belief) in generating a grasp. The results indicate that the human subjects, consciously, tried to use finger spread, the object's curves, and location of the robot hand relative to the object center of gravity to generate a good grasp. The effect of object weight, the robot wrist orientation being vertical or horizontal, and the ridges on the object surface in determining the grasp were also strong, but the subjects were not unanimous in using those heuristics. The subjects did not feel that they used the robot hand's palm heavily in generating the grasp. Finally, the subjects felt that their grasp strategy did not change during the experiment with practice.

\section{DISCUSSION}

The methods proposed by this paper (physical human interactive guidance, analysis using grasp measures, comparison with automated techniques) provide an exciting integration of the human physical experience and the human ability to extrapolate that experience to understand physical interaction in new scenarios along with the exhaustiveness of computer-based logic and simulation speed. Given the goals mentioned in Section I, our study has provided interesting results. It was, clearly, shown that the human-planned grasps performed well with a high success rate $(91 \%)$. While not near perfect like the human hand's grasping performance, the human-planned grasps were significantly better than the success rate of the state-of-the-art automated grasp planners (77\%, see Table IV).

Simultaneously, the physical interaction method showed that the human grasping method was similar in most aspects to a state-of-the-art automated grasp planner that, exhaustively, searched the entire configuration space for the best grasps. While the automated planner required hours across all objects to compute (suboptimal) grasps, the human subjects required only 5 min of practice to find the best grasps. This showed that humans excel at using their internal models to prune away large regions of the search space to, exponentially, speed up the search process. More work is also required in identifying why the existing grasping heuristics in the robotics community do not perform well when implemented on a physical robot as well as develop better heuristics for automated grasping.

This experiment also showed that the human subjects used grasp measures different from those used by automated grasp planners. The strong preference of humans to exploit an object's principal axes to perform a grasp even with a robotic hand prompted us to create a new grasp measure called skewness, which to our knowledge has not been mentioned previously in the literature. Our robot experiments showed that when skewness was used to filter the grasps from automated grasp planning, low-skewness GraspIt! grasps performed significantly better than high-skewness GraspIt! grasps.

Note that while we used the physical human interactive guidance method in a specific experimental setting with a chosen robot arm and hand combination and with objects placed in their natural configurations without clutter, our approach can easily be extended to other scenarios, such as using a different robot arm and hand, using cluttered environments where a direct approach to the object is unavailable, and using objects placed in non-natural orientations. It would interesting to see how the human-planned grasps and their performance would vary under other conditions.

\section{A. Robustness of Human-Planned Grasps}

Humans have a strong sense of causal physicality, or how objects in the physical world interact. Humans use this sense everyday when they interact with the world, specifically when they use tools to perform various tasks. Indeed, the human subjects may have considered the robotic hands that they used in the experiment as tools to perform the required task. The models of physical interaction that the subjects have internalized through their daily interactions would, certainly, have been used in conceiving the grasps to perform the various tasks [21], which would explain the higher quality of the human-planned grasps.

Even though the humans did not have the opportunity in this experiment to dynamically control grasp forces or finger location during the disturbance, which they typically do when performing grasps with their own hands [20], [45], just the geometry of low skewness provided significant performance benefits over automated grasp planning. The low skewness of wrist orientation in the human-planned grasps might seem obvious in hindsight, considering how a majority of everyday objects are designed with Cartesian coordinate frames. A grasp with low skewness provides two advantages with such "Cartesian" objects: 1) With Cartesian objects, palm contact and finger placement might be improved when the wrist orientation is parallel to or perpendicular to the object's principal axis. Since the BarrettHand had a flat palm, grasp with low skewness would, likely, generate more palm contact which created a more robust grasp. 
2) The contacts used in low-skewness grasps are more robust to small variations in contact location. For example, a grasp where the palm approached the soap bottle perpendicularly from the side is robust to small perturbations in position in the approach direction-all fingers will still have contact. In contrast, a grasp with high skewness that approached from the same side may lose contact at one finger due to a positioning error in the approach direction. This would cause the grasp to change from a three-finger contact to a weaker two-finger contact.

Indeed, humans might have a natural preference for grasps with low skewness, since human motor control literature has shown that many motor neurons encode human movements in extrinsic Cartesian coordinate frames rather than in intrinsic (muscle or joint) coordinate frames [23]. A deeper analysis of how skewness influence grasping performance, particularly, in different environment contexts (uncommon objects placed nonvertically in the presence of obstacles and clutter), will offer interesting insights into its effectiveness.

\section{B. Implications for Automated Grasp Synthesis}

While GraspIt! likely produced some of the best automated grasps, the mismatch between simulation models and the real world (in terms of, say, contact friction coefficients, unmodeled movement of the target object, and inaccurate soft-contact models) may have produced uncertainty in the grasping process and hurt the success rate of automated grasps. In addition, it could be that the automated grasp planners did not have the optimal grasp measures to narrow down on the best grasps.

One of the goals of this study is to use human skill to identify key grasp measures that can speed up automated grasp synthesis and improve real-world grasp quality. Table $\mathrm{V}$ shows that the skewness feature has significantly different values for humanplanned grasps and GraspIt! grasps. Furthermore, Fig. 7 shows that low-skewness grasps have significantly higher success rate than high-skewness grasps. These results indicate that an automated search process can focus on grasps with low skewness values before exploring grasps with higher skewness values. This will, likely, result in better grasps faster for GraspIt! and other automated grasp synthesis methods.

This paper did not further analyze the grasp measures that produced similar results between human-guided grasps and GraspIt!. This is because our data only contained highly successful grasps, and thus, it could not be used to identify good and bad grasp measures, unless significant differences were found between human-guided and GraspIt! grasps. In addition, the lack of correlation between epsilon and grasp wrench space volume with the high human-planned grasp success rates is worth investigating further to validate the grasp measures used by the grasping research community. In particular, a more rigorous experimental testing of these grasping heuristics is necessary.

\section{Task Dependence of Grasps}

An advantage of physical human interactive guidance is the simplicity with which the grasps that the human subjects specified for various tasks can be mapped into the robotic hand space. Previous studies have shown through human-subject ex- periments with datagloves, such as the Cyberglove ${ }^{7}$, that humans varied finger position carefully based on the task [16]. Indeed, it was also shown how finger posture influenced grasp force capability and stiffness. However, it is difficult to map the human-planned grasps to robot grasps.

In our study, the human subjects had an opportunity to control only finger and wrist placement (and not force and stiffness), and indeed, we saw some variability between tasks in finger posture (hand-flexion measure; see Table V). Specifically, for the coil of wire, the functional task was to "lift the object to remove a wire." It was noticed that the human subjects held the coil of wire by the rim, rather along its length as was the case in the lifting or handing over task. Similarly, for the CD pouch, the functional task was to hold the object so that it may be opened. These differences in grasps have been captured by the hand-flexion measure. However, we did expect to see more differences between the grasps for different tasks. We possibly need more appropriate grasp measures (than those measures listed in Table III) and object-task pairs that are suitable for differentiating task-specific human-planned grasping strategies. In addition, the large size of the robot hand relative to the object size could have influenced the human subjects to use similar grasps for the different tasks.

Interestingly, the human subjects chose grasps with low skewness independent of the task, indicating that humans valued a wrist configuration aligned with the object's principal axes significantly for grasping tasks. More work is required to understand wrist usage in grasping using the human hand.

\section{Human Grasping Heuristics}

Table VI presents a summary of the responses of the human subjects after performing the experiment. These responses provide insight into how the human subjects perceived their own actions and then enable us to compare the human subject's perception with a ground-truth measurement of their actions. It is clear that the subjects believed that object curves and using a spread-out finger configuration were critical aspects of the grasp. However, from Table V, we noticed that the human subjects tended to use reasonably small hand-spread values $\left(20^{\circ}\right.$ compared with $60^{\circ}$ for a equilateral-triangle grasp), indicating that they used the fingers closer to a parallel gripper form rather than a equilateral triangular grip.

Interestingly, even though low skewness was an important characteristic of the human-planned grasps, line 5 in Table VI showed that only $71 \%$ of the human subjects were conscious that the grasps that they performed had low skewness (we had expected a higher percentage). However, a more detailed study of the human-planned grasps with more subjects, more grasp measures, and machine learning techniques is necessary to derive insight into how humans plan grasps and manipulate objects in everyday life.

\footnotetext{
${ }^{7}$ http://www.cyberglovesystems.com/
} 


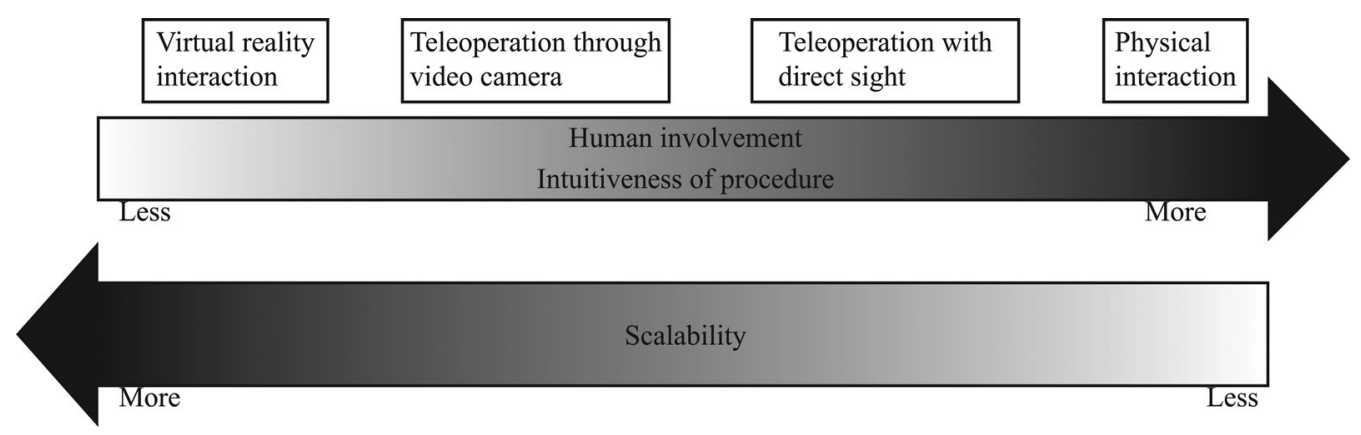

Fig. 8. Trade-offs in the procedure used to transfer human skills to robots.

\section{E. Improving Human-Subject Experiment Protocol}

When we were designing this experiment, we expected the human-planned grasps to have a success rate near $100 \%$; however, the human-planned grasps had a success rate of only $91 \%$. Why did the human-planned grasps have a success rate of only $91 \%$ ?

There might have been at least a few reasons related to the experiment protocol why the human-planned grasps had a lower success rate. First, we collected data from subjects who had never seen or interacted with a robotic arm/hand before. It is possible that with more practice with the robot, a subject would provide better grasping strategies.

Second, we asked human subjects to vary the grasping strategy every trial, if they could. In retrospect, we should not have forced the subjects to devise different grasping strategies as we do not believe that there are always multiple optimal solutions. Note, however, that there was one outlier in the human-planned lifting grasps success rates - the success rate for the 1-L botthe (only 40(13)\%, see Table IV). If this outlier is removed, the human grasping success rate is $97(1) \%$, even with vigorous shaking. As seen in Fig. 2, subjects chose to grasp the bottle from the top, when most humans with their own hand would not grasp a filled bottle this way. This strategy was chosen when we instructed subjects to vary the grasps when they could. This technique did not work well on the bottle's slippery surface and large mass.

Third, the subjects were not informed of the vigorous shaking used in the robustness test, and they only specified grasps for the various tasks. If the subjects had known about the shaking, they might have optimized their grasps for the shaking procedure. In contrast, the epsilon metric that GraspIt! uses actually optimizes the grasps for disturbances in all directions, similar to the disturbances in the shaking procedure. Thus, given that the human subjects did not know about the testing procedure, the human subjects were at a disadvantage compared with the GraspIt! grasps. However, the years of real-world experience still enabled the human subjects to perform better overall.

\section{F. Extent of Human Involvement}

In this experiment, the human subjects were involved heavily, guiding the robot hand, as though it was a child learning to perform a grasp. The human subject was provided to the robotic system information on where the wrist and fingers must be placed to perform a successful grasp. Surely, this information can be "taught" to a robotic system by other means as well, such as teleoperation or through interaction in virtual representation. Indeed, there are some advantages as the experiment moves toward using virtual representation - the experiment becomes increasingly scalable (see Fig. 8), since the experiment can be setup on a computer allowing the human subject to simply click where the robot should grasp the object instead of requiring a robotic arm and physical human involvement. Indeed, such supervised learning has been explored before [41].

However, these methods of using virtual representations to transfer human skill in physical interaction have only met with moderate success (60-80\% for multifingered grasping; see [41]) compared with the $91 \%$ success rate using physical human interactive guidance. In addition, the identification of new and powerful grasp measures (such as skewness) and human grasping principles has been rare through prior virtual methods.

The main challenge when using virtual experimental setups was that the task for the human became increasingly less intuitive as he/she moved away from physical interaction (see Fig. 8). Thus, the human-planned grasps might not be as effective the farther the human was removed from the task physically, since the specifics of the method (such as the view provided to the human) may interfere with his/her grasping method. More important than just the grasps the human provided, it might become harder to identify key grasping principles (such as skewness) when more virtualized experiment procedures are used. Thus, data fidelity might suffer with more virtualized experiment procedures despite their scalability. More work is required to identify the trade-off in scalability and data fidelity as the experiment procedure changes.

\section{CONCLUSION}

In this paper, we have shown that a novel experimental method called physical human interactive guidance can be used to obtain high-quality grasps planned by humans. The human-planned grasps were shown to be significantly better than grasps generated by state-of-the-art grasp planning algorithms (included in a program called GraspIt!). An elaborate grasp-measure set was also used to show that the human-planned grasps with the GraspIt! grasps were similar; however, a key contribution of this paper was to find a new grasp measure called skewness which 
explained why the GraspIt! grasps performed poorer than the human-planned grasps.

Finally, it was difficult to compare the performance of the physical interactive guidance method directly with other humanplanned grasping methods, because of the lack of available data in the literature. In this paper, we performed extensive experiments with a physical robot arm and hand to evaluate the grasp performance of the human-planned grasps as well as compared it with the state-of-the-art automated grasp planner. We look forward to comparing our results with results from other groups using different human-subject experiments for grasping.

\section{ACKNOWLEDGMENT}

The authors would like to thank B. Mayton for help with the robot experiment setup and L. LeGrand for interesting discussions on grasp metrics. The authors would also like to thank M. Ciocarlie and P. Allen of the GraspIt! team for help in the use of GraspIt! code.

\section{REFERENCES}

[1] R. Balasubramanian, L. Xu, P. Brook, J. R. Smith, and Y. Matsuoka, "Human-guided grasp measures improve grasp robustness on physical robot," in Proc. IEEE Int. Conf. Robot. Autom., 2010, pp. 2294-2301.

[2] Y. Bekiroglu, J. Laaksonen, J. Jorgensen, V. Kyrki, and D. Kragic, "Assessing grasp stability based on learning and haptic data," IEEE Trans. Robot., vol. 27, no. 3, pp. 616-629, Jun. 2011.

[3] G. M. Bone and Y. Du, "Multi-metric comparison of optimal 2d grasp planning algorithms," in Proc. IEEE Int. Conf. Robot. Autom., 2001, pp. 3061-3066.

[4] L. Y. Chang, R. L. Klatzky, and N. S. Pollard, "Selection criteria for preparatory object rotation in manual lifting actions," J. Motor Behav., vol. 42, no. 1, pp. 11-27, 2010.

[5] L. Y. Chang and Y. Matsuoka, "A kinematic thumb model for the act hand," in Proc. IEEE Int. Conf. Robot. Autom., 2006, pp. 1000-1005.

[6] L. Y. Chang and N. S. Pollard, "Constrained least-squares optimization for robust estimation of center of rotation," J. Biomech., vol. 40, pp. 1392 1400, 2007.

[7] E. Chinellato, A. Morales, R. B. Fisher, and A. P. del Pobil, "Visual quality measures for characterizing planar robot grasps," IEEE Trans. Syst., Man, Cybern. C, Appl. Rev., vol. 35, no. 1, pp. 30-41, Feb. 2005.

[8] A. Churchill, B. Hopkins, L. Rnnqvist, and S. Vogt, "Vision of the hand and environmental context in human prehension," Exp. Brain Res., vol. 134, pp. 81-89, 2000.

[9] M. T. Ciocarlie and P. K. Allen, "On-line interactive dexterous grasping," presented at the Eurohaptics, Madrid, Spain, 2008.

[10] S. T. Clanton, D. C. Wang, V. S. Chib, Y. Matsuoka, and G. D. Stetten, "Optical merger of direct vision with virtual images for scaled teleoperation," IEEE Trans. Vis. Comput. Graph., vol. 12, no. 2, pp. 277-285, Mar.-Apr. 2006.

[11] R. G. Cohen and D. A. Rosenbaum, "Where grasps are made reveals how grasps are planned: Generation and recall of motor plans," Exp. Brain Res., vol. 157, pp. 486-495, 2004.

[12] M. R. Cutkosky, "On grasp choice, grasp models, and the design of hands for manufacturing tasks," IEEE Trans. Robot. Autom., vol. 5, no. 3, pp. 269-279, Jun. 1989.

[13] R. Diankov and J. Kuffner, "OpenRAVE: A planning architecture for autonomous robotics," Robotics Institute, Carnegie Mellon Univ., Pittsburgh, PATech. Rep. CMU-RI-TR-08-34, Jul. 2008.

[14] C. Fernández, M. A. Vicente, C. Pérez, O. Reinoso, and R. Aracil, "Learning to grasp from examples in telerobotics," in Proc. IASTED Int. Conf. Artif. Intell. Appl., 2003, pp. 445-450.

[15] C. Ferrari and J. Canny., "Planning optimal grasps," in Proc. IEEE Int. Conf. Robot. Autom., 1992, pp. 2290-2295.

[16] J. Friedman and T. Flash, "Task-dependent selection of grasp kinematics and stiffness in human object manipulation," Cortex, vol. 43, pp. 444-460, 2007.
[17] S. S. H. U. Gamage and J. Lasenby. (2002, Jan.). "New least squares solutions for estimating the average centre of rotation and the axis of rotation," J. Biomech. [Online]. 35(1), pp. 87-93. Available: http://linkinghub.elsevier.com/retrieve/pii/S0021929001001609?showall $=\mathrm{t}$ rue

[18] C. Goldfeder, M. Ciocarlie, H. Dang, and P. Allen, "The Columbia grasp database," in Proc. Int. Conf. Robot. Autom., 2009, pp. 1710-1716.

[19] W. B. Griffin, R. P. Findley, M. L. Turner, and M. R. Cutkosky, "Calibration and mapping of a human hand for dexterous telemanipulation," in Proc. ASME IMECE Conf. Hapt. Interfaces Virt. Environ. Teleoperat. Syst. Symp., 2000.

[20] R. S. Johansson and G. Westling, "Roles of glabrous skin receptors and sensorimotor memory in automatic control of precision grip when lifting rougher or more slippery objects," Exp. Brain Res., vol. 56, no. 3, pp. 55064, 1984.

[21] S. H. Johnson-Frey, "What's so special about human tool use?," Neuron, vol. 39, pp. 201-204, 2003.

[22] L. A. Jones and S. J. Lederman, Human Hand Function. Oxford, U.K.: Oxford Univ. Press, 2006.

[23] S. Kakei, D. S. Hoffman, and P. L. Strick, "Muscle and movement representations in the primary motor cortex," Science, vol. 285, no. 5436, pp. 2136-2139, 1999.

[24] U. Kartoun, H. Stern, and Y. Edan, "Virtual reality telerobotic system," in Advances in E-Engineering and Digital Enterprise Technology-1. New York: Wiley, 2004.

[25] D. Kirkpatrick, B. Mishra, and C. K. Yap, "Quantitative Steinitz's theorems with applications to multifingered grasping," in Proc. ACM Symp. Theory Comput., 1990, pp. 341-351.

[26] C. Lee, "Learning reduced-dimension models of human actions," $\mathrm{Ph} . \mathrm{D}$ dissertation, The Robotics Institute, Carnegie Mellon University, Pittsburgh, PA, 2000.

[27] C. Lee and Y. Xu, "Reduced-dimension representations of human performance data for human-to-robot skill transfer," in Proc. IEEE Int. Conf. Robot. Autom., 1998, pp. 84-90.

[28] Z. Li and S. S. Sastry, "Task-oriented optimal grasping by multifingered robot hands," IEEE J. Robot. Autom., vol. 4, no. 1, pp. 32-44, Feb. 1988.

[29] J. Lloyd, J. Beis, D. Pai, and D. Lowe, "Model-based telerobotics with vision," in Proc. IEEE Conf. Robot. Autom., Apr. 1997, vol. 2, pp. 1297 1304.

[30] J. Lukos, C. Ansuini, and M. Santello, "Choice of contact points during multidigit grasping: Effect of predictability of object center of mass location," J. Neurosci., vol. 27, no. 4, pp. 3894-3903, 2007.

[31] A. Miller and P. K. Allen, "Graspit!: A versatile simulator for robotic grasping," in IEEE Robot. Autom. Mag., 2004.

[32] A. T. Miller and P. K. Allen, "Examples of 3D grasp quality computations," in Proc. IEEE Int. Conf. Robot. Autom., 1999, pp. 1240-1246.

[33] B. Mirtich and J. Canny, "Easily computable optimum grasps in 2-D and 3-D," in Proc. IEEE Int. Conf. Robot. Autom., 1994, pp. 739-747.

[34] N. Miyata, M. Kouchi, T. Kurihara, and M. Mochimaru, "Modeling of human hand link structure from optical motion capture data," in Proc. IEEE/RSJ Int. Conf. Intell. Robots Syst., 2004, pp. 2129-2135.

[35] A. Morales, E. Chinellato, A. Fagg, and A. del Pobil, "An active learning approach for assessing robot grasp reliability," in Proc. IEEE/RSJ Int Conf. Intell. Robots Syst., Sep. 28-Oct. 2, 2004, vol. 1, pp. 485-490.

[36] J. Ponce and B. Faveqon, "On computing three-finger force-closure grasps of polygonal objects," IEEE Trans. Robot. Autom., vol. 11, no. 6, pp. 868881, Dec. 1995.

[37] M. Ralph and M. Moussa, "An integrated system for user-adaptive robotic grasping," IEEE Trans. Robot., vol. 26, no. 4, pp. 698-709, Aug. 2010.

[38] J. Romano, K. Hsiao, G. Niemeyer, S. Chitta, and K. Kuchenbecker, "Human-inspired robotic grasp control with tactile sensing," IEEE Trans. Robot., vol. 27, no. 6, pp. 1067-1079, Dec. 2011.

[39] M. Santello, M. Flanders, and J. F. Soechting, "Postural hand synergies for tool use," J. Neurosci, vol. 18, no. 23, pp. 10105-10115, 1998 .

[40] A. Saxena, L. L. S. Wong, and A. Ng, "Learning grasp strategies with partial shape information," in Proc. 23rd Nat. Conf. Artif. intell. (AAAI'08), Chicago, Illinois, 2008, pp. 1491-1494.

[41] A. Saxena, J. Driemeyer, and A. Y. Ng, "Robotic grasping of novel objects using vision," Int. J. Robot. Res., vol. 27, no. 2, pp. 157-173, 2008.

[42] K. B. Shimoga, "Robot grasp synthesis algorithms: A survey," Int. J. Robot. Res., vol. 15, pp. 230-266, 1996.

[43] W. T. Townsend, "The BarrettHand grasper-programmably flexible part handling and assembly," Ind. Robot: Int. J., vol. 27, no. 3, pp. 181-188, 2000 . 
[44] M. Veber and T. Bajd, "Assessment of human hand kinematics," in Proc. IEEE Int. Conf. Robot. Autom., May 2006, pp. 2966-2971.

[45] G. Westling and R. Johansson, "Factors influencing the force control during precision grip," Exp. Brain Res., vol. 53, pp. 277-284, 1984.

[46] R. Wistort and J. R. Smith, "Electric field servoing for robotic manipulation," in Proc. IEEE/RSJ Int. Conf. Intell. Robots Syst., 2008, pp. 494-499.

[47] D. M. Wolpert, Z. Ghahramani, and M. I. Jordan, "Perceptual distortion contributes to the curvature of human reaching movements," Exp. Brain Res., vol. 98, pp. 153-156, 1994.

[48] V. M. Zatsiorsky and M. L. Latash, "Multifinger prehension: An overview," J. Motor Behav., vol. 40, no. 5, pp. 446-475, 2008.

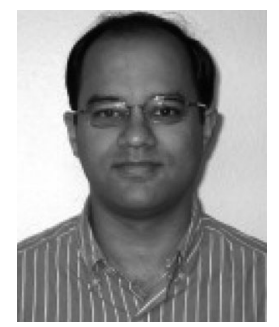

Ravi Balasubramanian received the B.Eng. and M.Eng. degrees in mechanical engineering from the National University of Singapore, Singapore, in 1999 and 2000, respectively, and the Ph.D. degree in robotics from Carnegie Mellon University, Pittsburgh, PA, in 2006.

He completed a Postdoctoral Fellowship in neurobotics with the University of Washington, Seattle, in 2009, and was a Research Scientist in mechanical engineering with Yale University until 2011. He is currently an Assistant Professor in mechanical, industrial, and manufacturing engineering with Oregon State University, Corvallis, where he directs the Robotics and Human Control Systems Laboratory. His research interests focus on manipulation and mobility and computational biomechanics and neuroscience.

Dr. Balasubramanian received the Best Student Paper Finalist Award at the IEEE International Conference on Robotics and Automation in 2004 for his doctoral research on a novel locomotion technique called legless locomotion.

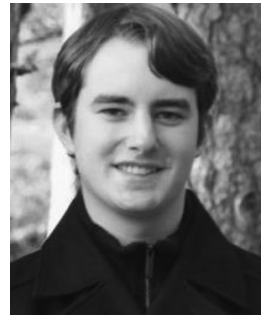

Peter D. Brook is currently working toward the B.S degree in computer science from the University of Washington, Seattle.

During his graduate study, his research focused on human-inspired and probabilistic methods for robotic grasp planning. He is currently a Software Engineer with Facebook, Menlo Park, CA, where he applies machine learning to tackle spam on the social graph.

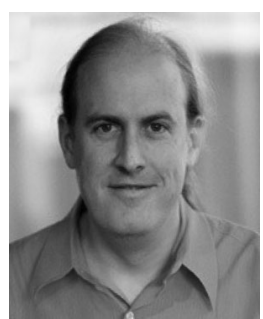

Joshua R. Smith received the B.A. degrees in computer science and in philosophy from Williams College, Williamstown, MA, in 1991, the M.A. degree in physics from Cambridge University, Cambridge, U.K., in 1997, and the S.M. and Ph.D. degrees from the MIT Media Lab, Cambridge, in 1995 and 1999, respectively.

He is currently an Associate Professor of electrical engineering and of computer science and engineering with the University of Washington (U.W.), Seattle, where he leads the Sensor Systems Research Group. At U.W., he leads the Communications and Interface Thrust, Center for Sensorimotor Neural Engineering, a National Science Foundation funded Engineering Research Center, and is a co-Principal Investigator of the Intel Science and Technology Center on Pervasive Computing. From 2004 to 2010, he was a Principal Engineer with Intel Labs, Seattle, where he founded and led the Personal Robotics project, as well as the Wireless Resonant Energy Link and Wireless Identification and Sensing Platform projects. He was the coinventor of an electric field sensing system for suppressing unsafe airbag firing that is included in every Honda car. His research interests include all aspects of sensor systems, including creating novel sensor systems, powering them wirelessly, and using them in applications such as robotics, ubiquitous computing, and human-computer interaction.

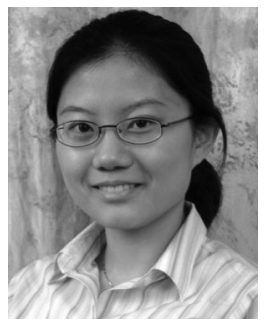

Ling Xu received the Bachelor's degree in computer science and the $\mathrm{Ph} . \mathrm{D}$. degree in robotics from Carnegie Mellon University, Pittsburgh, PA, in 2004 and 2011, respectively.

She worked for a year in industry before joining the Robotics Institute, Carnegie Mellon University, as a National Science Foundation Graduate Fellowship recipient. During her graduate career, her research was in the area of path planning for environmental coverage. More specifically, her thesis explored the application of ideas from graph theory and operations research toward a real-time path planner for optimal coverage of a space.

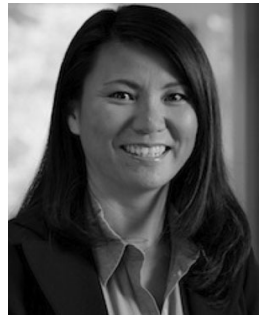

Yoky Matsuoka received the B.S. degree in electrical engineering and computer science (EECS) from the University of California, Berkeley, in 1993 and the M.S. and Ph.D. degrees in EECS from the Massachusetts Institute of Technology (MIT), Cambridge, in 1995 and 1998, respectively.

She was a Postdoctoral Fellow with the Brain and Cognitive Sciences Department, MIT, and in mechanical engineering with Harvard University. She is currently an Associate Professor with the Department of Computer Science and Engineering, University of

\section{Washington.}

Dr. Matsuoka received the Presidential Early Career Award for Scientists and Engineers in 2004, the IEEE Robotics and Automation Society Early Academic Career Award in 2005, and the MacArthur Fellowship in 2007. 